\title{
Using a Lay Cancer Screening Navigator to Increase Colorectal Cancer Screening Rates
}

\author{
Gerald Liu, MD, and Allen Perkins, MD, MPH
}

Introduction: Preventive care is often not performed during the ambulatory office visit due to the acute nature of the visit. One possible strategy is the use of a lay cancer screening navigator using the lay health worker model.

Methods: A training program for the lay cancer screening navigator and a patient registry for colorectal cancer screening was developed. The RE-AIM framework was used to evaluate the intervention. Descriptive statistics were generated for patient demographics.

Results: Reach: The lay cancer screening navigator contacted $91.9 \%$ of eligible patients. Effectiveness: At baseline, $28.6 \%$ of patients were current on their colorectal cancer screening, $40.5 \%$ at 6 months, and $42.2 \%$ at 12 months. Adoption: Patients contacted all reported being receptive to the intervention. Implementation: Of the 368 fecal immunochemical test kits mailed, 151 were returned (41.0\%), and $26(17.2 \%)$ were positive. Maintenance: The percentage of patients who were current between 6 months and at 12 months were not significantly different.

Discussion: This study demonstrates that the use of a lay cancer screening navigator to increase the rate of colorectal cancer screening is a viable strategy. ( $\mathrm{J}$ Am Board Fam Med 2015;28:280-282.)

Keywords: Cancer Screening, Colorectal Cancer, Prevention \& Control, Primary Health Care

Colorectal cancer remains a leading cause of cancer death despite effective screening modalities. Strategies to increase screenings include one-on-one interactions, patient reminders, and patient navigation. ${ }^{1}$ Use of navigation has not been found to be uniformly successful, often hampered by limited outreach success. $^{2,3}$ We examined the feasibility of using a lay health worker to increase colorectal cancer screening in a primary care residency practice.

\section{Methods}

The study was conducted from January 2013 to December 2013 in an urban, university-based Family Medicine residency.

Eligible patients included patients age 50 to 74 years seen in the clinic within the past 3 years who

This article was externally peer reviewed.

Submitted 20 July 2014; revised 11 August 2014; accepted 13 August 2014.

From the Department of Family Medicine, University of South Alabama, Mobile, AL.

Funding: This study was funded by the CDC through Grant No. C30117108.

Conflict of interest: none declared.

Corresponding author: Gerald Liu, MD, Department of Family Medicine, University of South Alabama, Mobile, AL 36604 (E-mail: gliu@southalabama.edu). were not at high risk for colorectal cancer, did not have a terminal disease, and were not part of special populations requiring an individualized approach. A lay cancer screening navigator was engaged and trained. The navigator made the initial contact by phone with a follow-up letter if no contact was made. Following contact and discussion, if the patient agreed to screening, the patient was offered either colonoscopy or a mailed fecal immunochemical test (FIT). If the FIT was not returned within 2 weeks, the navigator would call to remind the patient.

Data was collected at baseline, 6 months (immediately after the intervention), and at 12 months (6 months after the completion of the intervention). The primary outcome was the colorectal cancer screening rate. Demographics including the $\mathrm{Cu}-$ mulative Illness Rating Scale for Geriatrics (CIRS-G) were collected. ${ }^{4}$ The design was based on the RE-AIM framework. ${ }^{5}$

\section{Results}

There were 1394 eligible patients. At baseline, 398 (28.6\%) were current with colorectal cancer screening. As compared with those who were nor current, these patients were older $(P<.01)$ and had higher 
CIRS-G score $(P<.01)$. Insurance $(P<.01)$ and primary care provider $(P<.05)$ were also significant variables (Table 1). Reasons given for uncompleted screening included change in primary care provider, procrastination, lack of insurance, health literacy, cost, transportation, other medical issues, paranoia or fear, and a previous negative encounter.

\section{Reach}

During the intervention, the navigator made 915 calls, reaching $91.9 \%$ of the intervention population. Of those, $368(40.2 \%)$ agreed to colorectal cancer screening.

\section{Effectiveness}

At baseline, 28.6\% were current on colorectal cancer screening; immediately after the intervention, $40.5 \%$ of patients were current; and at 12 months (6 months after the intervention), $42.2 \%$ of patients were current. This was a significant change between baseline to 6 months $(P<.01)$, but not was not significant between 6 and 12 months $(P=.4)$. Of note, the majority of the completed screening was from an increase in the use of FIT.
Adoption

Patients contacted all reported being receptive to the intervention.

\section{Implementation}

Three hundred sixty-eight FITs were mailed, 151 were returned $(41.0 \%)$, and $26(17.2 \%)$ were positive requiring further testing.

\section{Maintenance}

The percentage of patients who were current between 6 months (immediately after the intervention) and at 12 months was not significantly different $(P=.4)$.

\section{Discussion}

This study demonstrates the feasibility of a lay cancer screening navigator to increase the rate of colorectal cancer screening. Most of the increase in colorectal cancer screening came from completion of FIT, which seemed to decrease barriers.

This study was likely successful because we were able to reach almost all eligible patients $(91.9 \%$ contacted). In addition, we offered an alternative method (FIT), which could be mailed to the patient. Impor-

Table 1. Comparison of Patient Demographics between Patients Who Are Current and Not Current on Colorectal Cancer Screening at Different Time Points

\begin{tabular}{|c|c|c|c|c|c|c|}
\hline \multirow[b]{2}{*}{ Characteristic } & \multicolumn{2}{|c|}{ Baseline } & \multicolumn{2}{|c|}{6 Months } & \multicolumn{2}{|c|}{12 Months } \\
\hline & Current & $\begin{array}{c}\text { Not } \\
\text { Current }\end{array}$ & Current & $\begin{array}{c}\text { Not } \\
\text { Current }\end{array}$ & Current & $\begin{array}{l}\text { Not } \\
\text { Current }\end{array}$ \\
\hline Total Number (\%) & $398(28.6)$ & $996(71.4)$ & $565(40.5)$ & $829(59.5)$ & $588(42.2)$ & $806(57.8)$ \\
\hline \multicolumn{7}{|l|}{ Screened with } \\
\hline Colonoscopy & $300(75.4)$ & & $347(61.4)$ & & $399(62.9)$ & \\
\hline FIT & $98(24.6)$ & & $218(38.6)$ & & $229(37.1)$ & \\
\hline Male (\% of total) & $133(33.4)$ & $327(32.8)$ & $179(31.7)$ & $281(33.9)$ & $181(30.8)$ & $372(34.7)$ \\
\hline Average age $\pm \mathrm{SD}^{*}$ & $60.3 \pm 6.0$ & $58.9 \pm 6.2$ & $60.3 \pm 6.0$ & $58.9 \pm 6.2$ & $60.4 \pm 6.0$ & $59.3 \pm 6.3$ \\
\hline Average CIRS $\pm \mathrm{SD}^{*}$ & $9.8 \pm 4.3$ & $8.7 \pm 3.9$ & $9.7 \pm 4.1$ & $8.6 \pm 3.9$ & $9.5 \pm 4.2$ & $8.6 \pm 4.0$ \\
\hline \multicolumn{7}{|l|}{ Insurance type $\mathrm{e}^{* \dagger}$} \\
\hline Commercial & 214 & 516 & 296 & 432 & 312 & 418 \\
\hline Medicare & 121 & 250 & 171 & 203 & 175 & 199 \\
\hline Medicaid & 55 & 199 & 86 & 167 & 87 & 164 \\
\hline None & 8 & 31 & 12 & 27 & 14 & 25 \\
\hline \multicolumn{7}{|l|}{$\mathrm{PCP}^{*}$} \\
\hline Faculty and NP & 296 & 581 & 393 & 481 & 410 & 465 \\
\hline Resident & 102 & 415 & 171 & 348 & 178 & 342 \\
\hline
\end{tabular}

${ }^{*} P<.05$ between current and not current comparisons at each time point.

${ }^{\dagger}$ The number of patients in each insurance type are different between time points due to movement between insurances (e.g., qualification for Medicare).

FIT, fecal immunochemical test; SD, standard deviation; CIRS, Cumulative Illness Rating Scale; PCP, primary care provider; NP, nurse practitioner. 
tant to our success was the development of an accurate patient registry. Multiple contacts outside of the visit enhanced the ability to overcome barriers, especially procrastination. Recruitment of the correct navigator seems to be critical.

In conclusion, use of a lay cancer screening navigator is a feasible strategy to increase the colorectal cancer screening rate in an underserved setting.

The authors thank Ms. Barbara Hodnett for her work as the lay cancer screening navigator on this project.

\section{References}

1. Holden DJ, Jonas DE, Porterfield DS, Reuland D, Harris R. Systematic Review: Enhancing the use and quality of colorectal cancer screening. Ann Intern Med 2010;152:668-676.

2. Leone LA, Reuland DS, Lewis CL, et al. Reach, Usage, and effectiveness of a medicaid patient navigator intervention to increase colorectal cancer screening, Cape Fear, North Carolina, 2011. Preventing Chronic Disease, 2013;10:E82.

3. Atlas SJ, Zai AH, Ashburner JM, et al. Non-visitbased cancer screening using a novel population management system. J Am Board Fam Med 2014;27: 474-485.

4. Miller MD, Towers A. A Manual of Guidelines for Scoring the Cumulative Illness Rating Scale for Geriatrics (CIRS-G). May 1991.

5. Glasgow RE, Vogt TM, Boles SM. Evaluating the public health impact of health promotion interventions: The RE-AIM framework. Am J Public Health 1999;89:1322-1327. 